JOURNAL OF

SYMPLECTIC GEOMETRY

Volume 6, Number 4, 379-405, 2008

\title{
SYMPLECTIC FIELD THEORY AND QUANTUM BACKGROUNDS
}

\author{
Michael Sullivan, John Terilla, and Thomas Tradler
}

We derive an $L_{\infty}$ structure associated to a polarized quantum background and characterize the obstructions to finding a versal solution to the quantum master equation (QME). We illustrate how symplectic field theory (SFT) is an example of a polarized quantum background and discuss the $L_{\infty}$ structure in the SFT context. The discussion may be summarized as follows: given a contact manifold $M$ with contact homology $\mathcal{H}$, one can define an $L_{\infty}$ algebra on $\mathcal{A}[[\hbar]]$, where $\mathcal{A}=\mathcal{A}(M)$ is the free symmetric algebra on the vector space of Reeb orbits of $M$. The obstructions to finding a versal solution to the QME in $\mathcal{A}[[\hbar]]$ are organized into what we call the kappa invariant, which is a new differential $\kappa: \mathcal{H}[[\hbar]] \rightarrow \mathcal{H}[[\hbar]]$.

Also, a quantum background associated to an arbitrary manifold is defined, which does not use any contact structure. It agrees with the one from SFT of the unit cotangent bundle of the manifold in some cases, but might, in general, be different.

\section{Introduction}

Since the celebrated Gromov compactness result for $J$-holomorphic curves in symplectic manifolds [7], symplectic geometers have been using these curves to study symplectic and contact manifolds, as well as questions in other fields connected to these manifolds. The compactness of these curves is often restated in some form of the equation

$$
\partial X=X * X
$$

Here $X$ is some collections of moduli spaces of these curves, $\partial$ is the topological boundary of these spaces, and $*$ embodies the (possibly self-) gluing of the $J$-holomorphic curves as they appear in Gromov's sequential limit. A common goal is to develop algebraic machinery (and its subsequent 
invariants) which can refine the equation $\partial X=X * X$. Current algebraic considerations include Floer homology, contact homology, and symplectic field theory (SFT) $[6]$.

Independently, efforts to understand in algebraic terms the way in which correlation functions are computed in the Batalin-Vilkovisky quantization have met with some success since the BV setting was generalized to quantum backgrounds. A quantum background consists of an algebraic framework within which a quantum master equation (QME) can be defined. According to Park $[\mathbf{8}, \mathbf{1 0}]$, the physicists' correlation functions are determined (up to finite ambiguity) by a versal solution to the QME.

In this paper, we note that the two areas are connected: SFT is an example of a quantum background. In fact, SFT is an example of a particularly nice kind of quantum background, which we call a polarized quantum background. For polarized quantum backgrounds, the QME has an expression in terms of an $L_{\infty}$ algebra, which we call the quantum $L_{\infty}$ algebra and is derived from the background data. For SFT, the quantum $L_{\infty}$ algebra is an invariant of the contact manifold. This $L_{\infty}$ structure contains (but is not contained in) all of the $L_{\infty}$ structures previously associated to a contact manifold. Augmentations, which are associated to symplectic fillings, are examples of solutions to the QME in this quantum $L_{\infty}$ algebra. The general theory of quantum backgrounds suggests that one should find, if possible, a versal solution to the QME, from which new contact invariants might be derived. There is a simple necessary and sufficient condition for the existence of a versal solution to the QME in a polarized quantum background, which we state for SFT in terms of $J$ holomorphic curves. Whether this condition is satisfied is itself a contact invariant. If the condition is not satisfied, there is a finer invariant which encodes the obstructions to the existence of a versal solution. This finer invariant, which we call the kappa invariant, is a differential $\kappa: \mathcal{H}[[\hbar]] \rightarrow \mathcal{H}[[\hbar]]$, where $\mathcal{H}$ is the contact homology of the contact manifold. While we develop $\kappa$ as the obstructions to solving the QME, the components of $\kappa=\kappa_{1} \hbar+\kappa_{2} \hbar^{2}+\cdots$ are linear maps on the contact homology that encode some kind of higher genus information. Since $\lim _{\hbar \rightarrow 0} \kappa=0$, the kappa invariant is invisible in the ordinary contact homology but can be seen clearly from the vantage of quantum backgrounds. Finally, we generalize an example of SFT $[\mathbf{3}]$ from surfaces to manifolds, using the involutive bi-Lie algebra structure of the manifold's string topology. We do not claim it to be SFT, but we confirm it defines a quantum background.

\section{Symplectic field theory}

We review the construction of SFT. For a more complete story, see [6]. 
A contact manifold is a pair $(M, \xi)$ where $M$ is a closed orientable $(2 n-1)$-dimensional manifold and $\xi \subset T M$ is a contact structure, that is, a $(2 n-2)$-dimensional maximally non-integrable distribution. By "maximally non-integrable" we mean that locally $\xi=\operatorname{ker}(\alpha)$ for some one-form $\alpha$ such that $\alpha \wedge(d \alpha)^{n}$ is nowhere-vanishing. If such an $\alpha$ is defined globally, then $\alpha$ is referred to as a contact one-form for $\xi$. Henceforth, assume such a form exists.

The Reeb vector field $R_{\alpha}$ is the unique vector field satisfying

$$
\alpha\left(R_{\alpha}\right)=1, \quad d \alpha\left(R_{\alpha}, \cdot\right) \equiv 0 .
$$

We usually denote the contact manifold simply as $M$ when it causes no ambiguity. Let $\mathcal{P}=\mathcal{P}(\alpha)$ denote the set of unparameterized Reeb orbits of $R_{\alpha}$ and their multiple covers. We only consider generic $\alpha$ whose Reeb orbits satisfy a certain non-degeneracy condition, although the so-called MorseBott set-up works as well [6]. To each orbit $\gamma \in \mathcal{P}$, one can associate the Conley-Zehnder index, $\mathrm{CZ}(\alpha)$, which captures a certain symplectic winding number of the linearized symplectic flow of $d \alpha \mid \xi$ about $\gamma$; See [6] for details. We ignore the issues of capping surfaces in this brief exposition.

For each $\gamma \in \mathcal{P}$, consider the two formal variables $p^{\gamma}$ and $q^{\gamma}$. We define a Weyl algebra $\mathcal{W}=\mathcal{W}(M)$ for the contact manifold to be the set of formal power series in $p$ and $\hbar$

$$
\sum_{\Gamma, g} f_{\Gamma, g}(q) p^{\Gamma} \hbar^{g}
$$

where $\Gamma=\left(\gamma_{1}, \ldots, \gamma_{a}\right)$ is a non-empty unordered $a$-tuple, $\gamma_{i} \in \mathcal{P}, g \in \mathbb{Z}$, $p^{\Gamma}=p^{\gamma_{1}} \cdots p^{\gamma_{a}}$, and $f_{\Gamma, g}(q)$ are polynomials in the $q$ variables.

All variables commute except $\left[p^{\gamma}, q^{\gamma}\right]=\kappa_{\gamma} \hbar$, where $\kappa_{\gamma}$ is the multiplicity of the orbit $\gamma$. Let $p^{\emptyset}$ and $q^{\emptyset}$ denote the unit 1 , which has grading 0 . The gradings of the other generators are

$$
\begin{aligned}
\left|p^{\gamma}\right| & =-\mathrm{CZ}(\gamma)+n-3, \\
\left|q^{\gamma}\right| & =\mathrm{CZ}(\gamma)+n-3, \\
|\hbar| & =2(n-3) .
\end{aligned}
$$

The symplectic manifold $\left(M \times \mathbb{R}_{t}, d\left(e^{t} \alpha\right)\right)$ is known as the symplectization of $(M, \xi)$. Fix an almost complex structure $J \in \operatorname{End}(T(M \times \mathbb{R}))$ with the following properties.

- Let $\tilde{\xi}$ be the extension of the distribution $\xi$ to $M \times \mathbb{R}$. Then $J(\tilde{\xi})=\tilde{\xi}$ and $J \mid \tilde{\xi}$ is $d\left(e^{t} \alpha\right)$-compatible: for each $p \in M \times \mathbb{R}$ and each $v, w \neq$ $0 \in T_{p}(M \times \mathbb{R}), d\left(e^{t} \alpha\right)\left(J_{p} v, J_{p} w\right)=d\left(e^{t} \alpha\right)(v, w)$ and $d\left(J_{p} v, v\right)>0$.

- $J$ is $\mathbb{R}$-invariant: $J_{(u, t)}=J_{(u, t+s)}$ for all $u \in M, s, t \in \mathbb{R}$.

- $J\left(\partial_{t}\right)=R_{\alpha}$, where $R_{\alpha}$ is the Reeb vector field extended to $M \times \mathbb{R}$.

Let $\Sigma_{g}(k, l)$ be a Riemann surface of genus $g$ with a set $\mathbf{x}_{+}$of $k$ "positively" marked punctures and a set $\mathbf{x}_{-}$of $l$ "negatively" marked punctures. Fix two 
collections of orbits, $\Gamma_{+}$and $\Gamma_{-}$. Let $\mathcal{M}_{g}\left(\Gamma_{+}, \Gamma_{-}\right)$be the moduli space of $(j, J)$-holomorphic maps (or $J$-holomorphic curves)

$$
(u, a): \Sigma_{g}(k, l) \rightarrow M \times \mathbb{R}
$$

with the property that at each puncture $x_{ \pm} \in \mathbf{x}_{ \pm}$, the map $a$ converges to $\pm \infty$ and the map $u$ converges to $\gamma_{ \pm} \in \Gamma_{ \pm}$. The convergence statement can be made more precise [6]. We mod out by the (possibly trivial) space of conformal reparameterizations of the domain $\left(\Sigma_{g}(k, l), j\right)$.

Note that most moduli spaces have a natural $\mathbb{R}$-translation, given by post-composing the curves with the shift $(u, a) \mapsto(u, a+t)$. One exception is the union of trivial cylinders which constitute $\mathcal{M}_{0}\left(\Gamma_{+}, \Gamma_{-}\right)$. Geometrically, these are simply copies of $\gamma \times \mathbb{R} \subset M \times \mathbb{R}$ with $\gamma \in \Gamma$. (To see that no other curves appear in $\mathcal{M}_{0}\left(\Gamma_{+}, \Gamma_{+}\right)$requires an application of Stokes' theorem as in Lemma 3.3 below.) For $\Gamma_{+} \neq \Gamma_{-}$, let $\hat{\mathcal{M}}_{g}\left(\Gamma_{+}, \Gamma_{-}\right)=\mathcal{M}_{g}\left(\Gamma_{+}, \Gamma_{-}\right) / \mathbb{R}$ denote the moduli space after modding out by this translation.

To each component of $\mathcal{M}_{g}\left(\Gamma_{+}, \Gamma_{-}\right)$, one can associate an element $A \in$ $H_{2}(M \times \mathbb{R})=H_{2}(M)$ by capping the image of any map in the component at the relevant orbits using certain capping surfaces. As in the Conley-Zehnder discussion, we ignore this issue. For those familiar with SFT, we mention that we are allowed to avoid this issue since for our examples of interest in Section 4, the first Chern class of the $J$-complex bundle restricted to $A$ vanishes: $c_{1}(J, A)=0$.

We denote by $|\Gamma|$ the cardinality of the set $\Gamma$. A widely accepted but still to be proven "fact" is that

Proposition 2.1. For generic $J, \mathcal{M}_{g}\left(\Gamma_{+}, \Gamma_{-}\right)$is an orbifold whose components are pre-compact in the sense of Gromov and have dimensions

$$
\sum_{\gamma \in \Gamma_{+}} \mathrm{CZ}(\gamma)-\sum_{\gamma \in \Gamma_{-}} \mathrm{CZ}(\gamma)+(n-3)\left(2-2 g-\left|\Gamma_{+}\right|-\left|\Gamma_{-}\right|\right)+2 c_{1}(J, A) .
$$

Write $\mathcal{M}^{k}$ as the set of $k$-dimensional components of $\mathcal{M}$. We define an element $H \in \mathcal{W}$ by counting the number of one-dimensional components of the moduli spaces

$$
H=\frac{1}{\hbar} \sum_{g, \Gamma_{+}, \Gamma_{-}} \frac{\left(\# \mathcal{M}_{g}^{1}\left(\Gamma_{+}, \Gamma_{-}\right)\right)}{\left|\Gamma_{+}\right| !\left|\Gamma_{-}\right| !} q^{\Gamma_{-}} p^{\Gamma_{+}} \hbar^{g} .
$$

Assuming $c_{1}(J, \cdot)=0$, note that the choice of the degrees of the elements implies that $H$ is an element of degree $(-1)$. Another widely accepted but still unproven "fact" is that $H^{2}=0$. Geometrically, $H^{2}$ is viewed as all possible ways of gluing $p^{\Gamma}$ in any term of $H$ to $q^{\Gamma}$ in any term of $H$, where $\Gamma$ is a (possibly empty) set of orbits. Technically, the proof of this equation would have two analytic steps: an application of Gromov compactness and a gluing theorem. We define $(\mathcal{W}, H)$ to be the SFT of $M$. 
If we only count holomorphic curves with genus $g=0$ and one positive puncture, $\left|\Gamma_{+}\right|=1$, our structure becomes a commutative differential graded algebra $(d, \mathcal{A})$, where $\mathcal{A}=S(V)$ is the symmetric algebra on the vector space $V$ generated by the $q^{\gamma}$ and $d$ is a degree -1 differential.

The homology of the DGA $(\mathcal{A}, d)$ is commonly known as contact homology. Note that we can write $d=\sum_{k=0} d_{k}$, where $d_{k}$ denotes the words of length $k$. Since $d_{0}$ may not be zero, this DGA is dual to a weak $L_{\infty}$ algebra. In case $d_{0}=0$, this weak $L_{\infty}$ structure is an $L_{\infty}$ structure and $d_{1}: V \rightarrow V$ satisfies $d_{1}^{2}=0$. (This is revisited in Section 3.4, where we call the structure the level zero $L_{\infty}$ algebra.) The $d_{1}$ homology of $V$, when defined, is called the linearized, or cylindrical, contact homology.

The construction of the SFT of $M$ depends on the choice of contact oneform $\alpha$ and almost complex structure $J$. This motivates one more concept. A directed symplectic cobordism from the contact manifold $\left(M_{-}, \xi_{-}\right)$with contact form $\alpha_{-}$and almost complex structure $J_{-}$to the contact manifold $\left(M_{+}, \xi_{+}\right)$with contact form $\alpha_{+}$and almost complex structure $J_{+}$is a symplectic manifold $(C, \omega)$ and $\omega$-compatible complex structure $J \in \operatorname{End}(T C)$ with the following properties.

- There exist (possibly empty) neighborhoods $C_{-}, C_{+}$in $C$ such that $C \backslash\left(C_{-} \cup C_{+}\right)$is compact.

- $\left(C_{ \pm}, \omega \mid C_{ \pm}\right)$is symplectomorphic to a neighborhood of the \pm -end of the symplectization of $M_{ \pm}$. Moreover, this symplectomorphism carries $J$ to $J_{ \pm}$.

We say the cobordism is exact if $\omega$ is exact. Exact symplectic cobordisms, such as symplectizations, enjoy the property that the moduli space of holomorphic curves without punctures is empty

We associate to $C$ the graded commutative algebra $D=D\left(C, \alpha_{ \pm}\right)$consisting of power series

$$
\sum_{\Gamma_{+}, g} \phi_{\Gamma_{+}, g}\left(q^{-}\right)\left(p^{+}\right)^{\Gamma+} \hbar^{g},
$$

where $\Gamma_{+}=\left(\gamma_{1}, \ldots, \gamma_{a}\right)$ is a non-empty unordered $a$-tuple, $\gamma_{i} \in \mathcal{P}\left(\alpha_{+}\right)$, $g \in \mathbb{Z}, p^{\Gamma_{+}}=\left(p^{+}\right)^{\gamma_{1}} \cdots\left(p^{+}\right)^{\gamma_{a}}$, and $\phi_{\Gamma_{+}, g}\left(q^{-}\right)$are polynomials in the $q^{-}$ variables. All variables commute.

Given sets of $\left(\alpha_{ \pm}\right)$-Reeb orbits $\Gamma_{ \pm}$, let $\mathcal{M}_{g}\left(\Gamma_{+}, \Gamma_{-}\right)$denote the moduli space of $J$-holomorphic curves in $C$ with asymptotic behavior at the positive/negative punctures. Note that in this case the moduli space does not have the $\mathbb{R}$-translation. Define the element $F \in \frac{1}{\hbar} D$ to count the zerodimensional components of the moduli spaces

$$
F=\frac{1}{\hbar} \sum_{g, \Gamma_{+}, \Gamma_{-}}\left(\# \mathcal{M}_{g}^{0}\left(\Gamma_{+}, \Gamma_{-}\right)\right)\left(q^{-}\right)^{\Gamma_{-}}\left(p^{+}\right)^{\Gamma_{+}} \hbar^{g} .
$$

Such an element is called a potential of $C$. 
Define a left action of $\mathcal{W}^{-}$on $D$ by considering "full-gluing". More precisely, if $p_{\gamma}^{-} \in \mathcal{W}^{-}$, define its left action on $D$ using the substitution

$$
p_{\gamma}^{-} \mapsto \kappa_{\gamma} \hbar \frac{\vec{\partial}}{\partial q_{\gamma}^{-}} .
$$

Define a similar left action of $\mathcal{W}^{+}$on $D$. The analogue of $H^{2}=0$ is $\vec{H} e^{F}=e^{F} \overleftarrow{H}$

Let $f$ be the count of holomorphic curves in $C$ with genus $g=0$ and one positive puncture $\left|\Gamma_{+}\right|=1$, then this count defines a DGA homomorphism $f_{*}$ from $\mathcal{A}_{+}$to $\mathcal{A}_{-}$. If $M_{+}$and $M_{-}$are contactomorphic, another unproven but commonly assumed "fact" is that a directed exact symplectic cobordism can be chosen such that the induced map $f_{*}$ is a quasi-isomorphism.

\section{Quantum backgrounds}

In this section, we introduce quantum backgrounds and polarized backgrounds, relating them to the original concepts from physics. We derive several $L_{\infty}$-structures for polarized quantum backgrounds. In any quantum background, one can speak about a QME, but in the polarized case, the QME has an expression in terms of one of the derived $L_{\infty}$ algebras. We discuss a complete obstruction to the existence of a versal solution to the QME. Throughout the section, we translate these concepts to SFT, which is an example of a polarized quantum background.

Definition 3.1. Let $k$ be a field of characteristic zero. We say that a graded associative, unital $k[[\hbar]]$ algebra $W$ has a classical limit provided $W$ is free as a $k[[\hbar]]$ module and if $K=W / \hbar W$ is a graded, commutative, associative, unital $k$ algebra. $K$ is called the classical limit.

If an algebra $W$ has a classical limit, then $W \simeq K \oplus \hbar K \oplus \hbar^{2} K \oplus \cdots$ and $[W, W] \subseteq \hbar W$. The Weyl algebra $W=\mathcal{W}(M)$ associated to a contact manifold $M$ is an example of an algebra with a classical limit. The classical limit of $\mathcal{W}(M)$ is the commutative ring $K=S(V)\left[\left[V^{*}\right]\right]$, where $V$ is the graded vector space generated by the Reeb orbits $\mathcal{P}$ and $S(V)$ is the symmetric algebra of $V$. This classical limit is like the graded commutative algebra underlying the differential Poisson algebra in $[6]$.

Definition 3.2. We define a (quantum) background $B$ to be a four-tuple $B=(W, H, N, \varphi)$, where

(1) $W=\oplus_{i} W^{i}$ is a $k[[\hbar]]$ algebra with a classical limit;

(2) $H \in W^{1}$ satisfies $H^{2}=0$ ( $H$ is called a structure);

(3) $N=\oplus_{i} N^{i}$ is a graded left $W$ module, which is free as a $k[[\hbar]]$ module;

(4) $\varphi \in N^{0}$ satisfies $H \cdot \varphi=0$ ( $\varphi$ is called a vacuum). 
A morphism between $B=(W, H, N, \varphi)$ and $B^{\prime}=\left(W^{\prime}, H^{\prime}, N^{\prime}, \varphi^{\prime}\right)$ consists of a map $\sigma: W \rightarrow W^{\prime}$ of graded $k[[\hbar]]$ algebras and a map $\tau: N \rightarrow N^{\prime}$ of graded $W$ modules (where $N^{\prime}$ becomes a $W$ module via $\sigma$ ) with $\tau(\varphi)=\varphi^{\prime}$ satisfying the compatibility

$$
\tau(H a \varphi)=H^{\prime} \sigma(a) \varphi^{\prime} \text { for all } a \in W .
$$

Let us motivate the definition of a quantum background by recalling how similar concepts arise in physics. In the Batalin-Vilkovisky approach to quantum field theory, an action functional is a function of fields $S$ that satisfies the so-called QME $\Delta\left(e^{S / \hbar}\right)=0$. As one would expect, the deformations of the action functional give invariants of the field theory, which have expressions in the physics language as well as the language of deformation theory. For example, the space of infinitesimal deformations of the action corresponds to the space of physical observables. One may qualify the observables by calling them quantum observables to distinguish them from their $\hbar=0$ limits, which are the classical observables. On the deformation theory side, these are characterized by two homology theories: one with $\hbar$ 's, one without. Back on the physics side, there are physical invariants associated to a collection of observables called correlation functions. They are typically derived by physicists with the computational aid of a Feynman path integral, but can be extracted from a versal deformation of the action functional, provided it exists. In the deformation theory language, the correlation functions are captured by a kind of flat connection on the tangent bundle of the moduli space of solutions to the QME [10]. Thus, the process of beginning with a physical quantum field theory and producing the correlation functions of the theory can be recast mathematically in the language of deformation theory as producing a versal solution to the QME and deriving some algebraic structures. So, in practical terms, if one is in a setting where a QME can be defined, an approach to extracting invariants is to try and compute correlation-function type invariants, with the first step of seeking a versal solution to the QME.

In certain situations, and guided by general principles of homotopy algebra, the algebraic setting in which a QME can be defined should be generalized beyond that of Batalin-Vilkovisky algebras. Quantum backgrounds provide a more general algebraic background setting in which fields, action functionals, master equations, etc. can be defined [8, 10]. If $(W, H, N, \varphi)$ is a quantum background, the QME for an element $S \in W$ is the equation $e^{-S / \hbar} H e^{S / \hbar} \varphi=0$. The following is a translation into physics terminology of the components: the module $N$ is the space of the fields, the ring $W$ consists of functionals on the fields, the structure $H$ is a package containing the BRST and Batalin-Vilkovisky differentials and their higher order analogs, and the vacuum $\varphi$ is (the exponential of) an initial action functional applied to some vacuum field. The factor of $e^{-S / \hbar}$ on the left side of 
should be thought of as a way to control the $\hbar$ so the result does not have any negative powers of $\hbar$.

SFT is precisely an example of a quantum background. As such, one can think of the data afforded by SFT as providing a mathematical context in which one can speak of a quantum action functional, specifically as an element of $W$ satisfying a QME. The quantum background arising from SFT comes equipped with zero as an initial action functional (that zero is an action functional is a non-trivial condition and follows from Lemma 3.3). Then, the physical considerations mentioned above suggest a way to process SFT: find, if possible, a versal solution to the QME in the SFT quantum background. In principle, the physically inspired invariants which are encoded in this versal solution - or the obstructions to the existence of such a versal solution - give invariants of the underlying SFT. It should be acknowledged that SFT has roots in the $A$ model topological field theory, which itself is a kind of field theory. Perhaps one can think of SFT in its current form of a square zero element in the Weyl algebra, which goes beyond the $A$ model origins, as a theory primed for a "second" quantization, the first step of which is to study the QME in the quantum background defined by SFT.

3.1. SFT is an example of a quantum background. Consider the Weyl algebra $\mathcal{W}(M)$ of a contact manifold $M$. Recall that $\mathcal{A}$ is the symmetric algebra generated by the Reeb orbits, that is, polynomials in the " $q$ " variables. Notice that $\mathcal{A}[[\hbar]]$ is a left $\mathcal{W}$ module with the module action $\mathcal{W} \otimes_{k[[\hbar]]} \mathcal{A}[[\hbar]] \rightarrow \mathcal{A}[[\hbar]]$ defined by

$$
f(q) p_{\gamma_{1}} \cdots p_{\gamma_{a}} \cdot g(q) \mapsto f(q)\left(\hbar \frac{\partial}{\partial q_{\gamma_{1}}}\right) \cdots\left(\hbar \frac{\partial}{\partial q_{\gamma_{a}}}\right) g(q) .
$$

Let $H$ be defined as in equation (2.1), but without the overall factor of $\frac{1}{\hbar}$. This does not affect the fact that the geometry of moduli spaces of $J$-holomorphic curves implies $H^{2}=0$. We do not use the SFT convention of putting the $\frac{1}{\hbar}$ factor in front of $H$, since for a quantum background, $H$ should be an element of the ring $W$, and $\frac{1}{\hbar} H \notin W$. The following lemma implies that $H \cdot 1=0$ for $1 \in \mathcal{A}[[\hbar]]$.

Lemma 3.3. Each monomial in $H$ has a factor of $p$.

Proof. We recall this standard SFT fact. If $p^{\Gamma_{+}} q^{\Gamma_{-}} \hbar^{g-1}$ is a term in $H$, there exists $u \in \mathcal{M}_{g}^{1}\left(\Gamma_{+}, \Gamma_{-}\right)$. Since $J$ is $d\left(e^{t} \alpha\right)$-compatible, $\int u^{*} d \alpha$ is positive; thus, by the Stokes' theorem,

$$
0<\int u^{*} d \alpha=\int_{\Gamma_{+}} \alpha-\int_{\Gamma_{-}} \alpha .
$$

Since all the integrals on the RHS are positive, $\Gamma_{+} \neq \emptyset$. 
This lemma shows that $H \cdot 1=0$. Therefore, the four-tuple

$$
(\mathcal{W}(M), H, \mathcal{A}[[\hbar]], 1)
$$

forms a quantum background.

3.2. Algebraic structures on the module. Before turning to the QME and the problem of finding a versal solution, we discuss a special feature of the background arising from SFT that can be used to equip the module with several algebraic structures. We do this because the QME has a simple restatement using this algebraic structure.

To explain this, it is convenient to have another picture of the action of $\mathcal{W}$ on $\mathcal{A}[[\hbar]]$. Consider the inclusion $\iota: \mathcal{A}[[\hbar]] \rightarrow \mathcal{W}$ defined by viewing a polynomial in $q$ and $\hbar$ as an element of $\mathcal{W}$, and the projection pr: $\mathcal{W} \rightarrow$ $\mathcal{A}[[\hbar]]$ defined by setting the $p$ 's to zero after writing an element in canonical form with $q$ 's on the left. The action of $\mathcal{W}$ on $\mathcal{A}[[\hbar]]$ can be viewed as

$$
\left(f(q) p_{\gamma_{1}} \cdots p_{\gamma_{a}}\right) \cdot g(q) \mapsto \operatorname{pr}\left[f(q) p_{\gamma_{1}} \cdots p_{\gamma_{a}}, \iota(g(q))\right],
$$

where [,] is the graded commutator in $\mathcal{W}$. Backgrounds $(W, H, N, \varphi)$, for which the $W$ module structure on $N$ can be understood using an inclusion $\iota: N \rightarrow W$ of $N$ as a commutative subalgebra of $W$ and a projection pr: $W \rightarrow N$, naturally have additional algebraic structures on $N$.

Definition 3.4. Let $B=(W, H, N, \varphi)$ be a background and suppose $N=$ $W / I$ is the quotient of $W$ by a left ideal $I$ and the left $W$ module structure on $N$ is the one obtained by left multiplication. We say that $B$ is polarized by $\iota: N \rightarrow W$ if $\iota$ includes $N$ as a commutative $k[[\hbar]]$ subalgebra of $W$. We call $B=(W, H, N, \varphi, \iota)$ a polarized background.

We first describe two algebraic structures on the module of a polarized quantum background: one is an $L_{\infty}$ algebra over $k[[\hbar]]$ called the quantum $L_{\infty}$ structure, another is an $L_{\infty}$ algebra over $k$ called the classical $L_{\infty}$ structure. Suppose that $(W, H, N, \varphi, \iota)$ is a polarized quantum background. Here $N=W / I$, and there is a natural projection pr $: W \rightarrow N$. Define multilinear operators $\mu_{k}: N^{\times k} \rightarrow N$ by

$$
\mu_{k}\left(g_{1}, \ldots, g_{k}\right)=\operatorname{pr}\left(\frac{1}{\hbar^{k}}\left[\cdots\left[H, \iota\left(g_{1}\right)\right], \ldots, \iota\left(g_{k}\right)\right]\right) .
$$

Note that $[W, W] \subseteq \hbar W$ implies that $\left[\cdots\left[H, \iota\left(g_{1}\right)\right], \ldots, \iota\left(g_{k}\right)\right]$ is divisible by $\hbar^{k}$ and so the $\mu_{k}$ are well defined. (If one were to use an overall factor of $\frac{1}{\hbar}$ in front of $H$, then one should divide by $\hbar^{k-1}$, instead of $\hbar^{k}$, in the definition of $\mu_{k}$.)

Theorem 3.5. If $B=(W, H, N, \varphi, \iota)$ is a polarized quantum background, then $\left(N,\left\{\mu_{k}\right\}\right)$ is an $L_{\infty}$ algebra over $k[[\hbar]]$. 
Proof. First, we show that the $\mu_{k}$ are symmetric. To see this, observe that the terms in $\left[\cdots\left[H, \iota\left(g_{1}\right)\right], \ldots, \iota\left(g_{k}\right)\right]$ are exactly those of the form

$$
\iota\left(g_{\sigma(1)}\right) \cdots \iota\left(g_{\sigma(l)}\right) H \iota\left(g_{\sigma(l+1)}\right) \cdots \iota\left(g_{\sigma(k)}\right),
$$

where $\sigma$ is a permutation satisfying $\sigma(1)<\cdots<\sigma(l)$ and $\sigma(l+1)<\cdots<$ $\sigma(k)$. Because $\iota(N)$ is a commutative subalgebra of $P$, we can reorder all the factors that precede $H$ and all the factors the follow $H$. For example, replacing $\iota\left(g_{i}\right) \iota\left(g_{i+1}\right)$ by $(-1)^{\left|g_{i}\right|\left|g_{i+1}\right|} \iota\left(g_{i+1}\right) \iota\left(g_{i}\right)$ in every term it appears gives $\mu_{k}\left(g_{1}, \ldots, g_{i}, g_{i+1}, \ldots, g_{k}\right)=(-1)^{\left|g_{i}\right|\left|g_{i+1}\right|} \mu_{k}\left(g_{1}, \ldots, g_{i+1}, g_{i}, \ldots, g_{k}\right)$, as needed.

To see that the $L_{\infty}$ relations are satisfied by the $\mu_{k}$, choose any homogeneous elements $g_{1}, \ldots, g_{k} \in N$ and consider the ring $R=k[[\hbar]]\left[t_{1}, \ldots, t_{k}\right]$, where $\left|t_{i}\right|=-\left|g_{i}\right|$. Let $F=\sum_{i=1}^{k} g_{i} t_{i}$ and consider $e^{F / \hbar} H e^{-F / \hbar}$ :

$$
\begin{aligned}
e^{F / \hbar} H e^{-F / \hbar} & =H+\frac{1}{\hbar}[H, F]+\frac{1}{2 ! \hbar^{2}}[[H, F], F]+\frac{1}{3 ! \hbar^{3}}[[[H, F], F], F]+\cdots \\
& =H+\sum_{j=1}^{\infty} \frac{1}{j !} \mu_{j}(F, \ldots, F) .
\end{aligned}
$$

Since, $H^{2}=0 \Rightarrow\left(e^{F / \hbar} H e^{-F / \hbar}\right)^{2}=0$. In particular, the coefficient of the $t_{1} \cdots t_{k}$ term in $\left(e^{F / \hbar} H e^{-F / \hbar}\right)^{2}$ vanishes. Explicitly,

$$
\sum_{\sigma, i} \chi(\sigma)(-1)^{(i)(k-i)} \mu_{i}\left(f_{\sigma(1)}, \ldots, f_{\sigma(i-1)}, \mu_{k-i}\left(f_{\sigma(i)}, \ldots, f_{\sigma(k)}\right)\right)=0,
$$

where the sum is over all $k$-permutations $\sigma$ satisfying $\sigma(1)<\cdots<\sigma(i-1)$ and $\sigma(i)<\cdots<\sigma(k)$ and $\chi(\sigma)$ is the Koszul sign of $\sigma$, and over all $i=$ $1, \ldots, k-1$. Equation (3.1) is precisely the $L_{\infty}$ relations for the $\left\{\mu_{k}\right\}$.

The module $N$ is a free $k[[\hbar]]$ module. If we write $N$ as $N=\mathcal{A}[[\hbar]]$, then each operator $\mu_{k}: N^{k} \rightarrow N$ can be decomposed in its $\hbar$ powers as

$$
\mu_{k}=\mu_{k 0}+\hbar \mu_{k 1}+\hbar^{2} \mu_{k 2}+\cdots
$$

with each $\mu_{k i}: \mathcal{A}^{k} \rightarrow \mathcal{A}$. Expressing equation (3.1) in powers of $\hbar$ implies, in particular, that the $\left(\mathcal{A},\left\{\mu_{k 0}\right\}\right)$ form an $L_{\infty}$ algebra over the field $k$.

Definition 3.6. Let $B=(W, H, N, \varphi, \iota)$ be a polarized background. Let $\mathcal{A}=\left.N\right|_{\hbar=0}$. We call the algebra $\left(\mathcal{A}[[\hbar]],\left\{\mu_{k}\right\}\right)$ the quantum $L_{\infty}$ algebra associated to $B$ and we call $\left(\mathcal{A},\left\{\mu_{k 0}\right\}\right)$ the classical $L_{\infty}$ algebra associated to $B$.

3.3. Example: differential BV algebras. In [3], Cieliebak and Latschev describe a square zero element in the Weyl algebra as affording a kind of BV infinity structure. The operator $D$ defined in $[\mathbf{3}]$ is the same as $\mu_{1}$. While we think of this structure in different terms, certainly differential BV algebras provide simple, nontrivial examples with which to illustrate our 
constructions. Suppose that $V$ is a graded vector space with basis $\left\{q^{i}\right\}$. Let $\left\{p_{i}\right\}$ be the dual basis for $V^{*}$ and consider the Weyl algebra $\mathcal{W}$ consisting of power series in the variables $p_{i}$ and $\hbar$ with coefficients that are polynomials in the $q^{i}$. We have the commutation relations $\left[q^{i}, q^{j}\right]=\left[p_{i}, p_{j}\right]=0$ and $\left[p_{i}, q^{j}\right]=\hbar \delta_{i}^{j}$. Suppose that

$$
H=\sum_{i} f^{i}(q) p_{i}+\sum_{i j} f^{i j}(q) p_{i} p_{j}
$$

is odd degree, square zero, and $f^{i}(q), f^{i j}(q) \in S(V)=\mathcal{A}$. Then $(\mathcal{W}, H, \mathcal{A}[[\hbar]], 1, \iota)$ is a polarized quantum background. Here, $\mathcal{A}[[\hbar]]$ is the quotient of $\mathcal{W}$ by the left ideal $I$ generated by the $p$ 's, and $\iota: \mathcal{A}[[\hbar]] \rightarrow \mathcal{W}$ is the inclusion.

Let us analyze the quantum $L_{\infty}$ structure $\left(\mathcal{A}[[\hbar]],\left\{\mu_{k}\right\}\right)$. Since $H$ is quadratic in $p, \mu_{k}=0$ for $k>2$. The operators $\mu_{1}=\mu_{10}+\hbar \mu_{11}$ and $\mu_{2}=\mu_{20}$ are given by

$$
\mu_{1}(g(q))=\sum_{i} f^{i}(q) \frac{\partial g}{\partial q^{i}}+\hbar \sum_{i j} f^{i j}(q) \frac{\partial^{2} g}{\partial q^{i} q^{j}}
$$

and

$$
\mu_{2}\left(g_{1}(q), g_{2}(q)\right)=\sum_{i j} f^{i j}(q) \frac{\partial g_{1}}{\partial q^{i}} \frac{\partial g_{2}}{\partial q^{j}} .
$$

Write $\mu_{10}, \mu_{11}$, and $\mu_{20}$ as $d, \Delta$, and $\{$,$\} respectively. Then the$ quantum and the classical $L_{\infty}$ algebras associated to the background $(\mathcal{W}, H, \mathcal{A}[[\hbar]], 1, \iota)$ are the differential graded Lie algebras:

$$
\text { quantum dgLa }=(\mathcal{A}[[\hbar]], d+\hbar \Delta,\{,\})
$$

and

$$
\text { classical dgLa }=(\mathcal{A}, d,\{,\}) .
$$

Note that $H^{2}=0$ implies that $d^{2}=d \Delta+\Delta d=\Delta^{2}=0$. The triple $(\mathcal{A}, d, \Delta)$ defines a differential BV algebra and the Lie bracket $\mu_{20}$ is the one defined by the failure of $\Delta$ to be a derivation of the commutative, associative product in $\mathcal{A}$. Theorem 3.5 is suggestive of the process of deriving a Lie algebra structure from a BV algebra, but the quantum $L_{\infty}$ algebra is $\hbar$-sensitive in a crucial way. To see the importance of the role of $\hbar$, we make a simple comparison between the two dgLas $(\mathcal{A}, d+\Delta,\{\}$,$) and (\mathcal{A}[[\hbar]], d+\hbar \Delta,\{\}$,$) .$ Since $\{a, b\}=(d+\Delta)(a \cdot b)$ for two closed $a, b$, the bracket vanishes in the homology of $(V, d+\Delta,\{\}$,$) . However, the brackets in (\mathcal{A}[[\hbar]], d+\hbar \Delta,\{\}$, do not, in general, vanish in homology - if $a$ and $b$ are $(d+\hbar \Delta)$-closed, 
then $(d+\hbar \Delta)(a \cdot b)=\hbar\{a, b\}$, but since $\hbar$ is not a unit in $\mathcal{A}[[\hbar]]$, it may be that $\{a, b\}$ is not exact.

3.4. The quantum $L_{\infty}$ structure from SFT. It is interesting to calculate the quantum $L_{\infty}$ structure arising from SFT and express it directly in terms of moduli spaces of $J$-holomorphic curves.

Proposition 3.7. For $k=1, \ldots, i$, let $\Gamma_{k}$ be a non-empty set of orbits. Then

$$
\begin{aligned}
\mu_{i j}\left(q^{\Gamma_{1}}, \ldots, q^{\Gamma_{i}}\right)= & \sum_{q^{\Gamma \in \mathcal{A}}} \sum_{k=i}^{i+j} \sum_{\substack{\gamma_{1} \in \Gamma_{1}, \ldots, \gamma_{i} \in \Gamma_{i} \\
\left\{\gamma_{1}, \ldots, \gamma_{k}\right\} \subset\left(\Gamma_{1} \cup \cdots \cup \Gamma_{i}\right)}} \\
& \#\left(\mathcal{M}_{i+j-k}^{1}\left(\left\{\gamma_{1}, \ldots, \gamma_{k}\right\}, \Gamma\right)\right) q^{\Gamma} q^{\left(\Gamma_{1} \cup \ldots \cup \Gamma_{i}\right) \backslash\left\{\gamma_{1}, \ldots, \gamma_{k}\right\}},
\end{aligned}
$$

where the sets of orbits can have repeated elements.

Proof. We first note that the exponent of $\hbar$ in the contribution of $\mu_{i j}\left(q^{\Gamma_{1}}, \ldots\right.$, $\left.q^{\Gamma_{i}}\right)$ to the expression $\left[\cdots\left[H, q^{\Gamma_{1}}\right], \ldots, q^{\Gamma_{i}}\right]$ is both $i+j$ and $g+k$, where $g$ is the genus of the holomorphic curve and $k$ is the number of positive punctures. Thus, $g=i+j-k$, as written.

For the moduli space not to contribute to a vanishing term when we set the $p$ variables equal to 0 , we require that $\left\{\gamma_{1}, \ldots, \gamma_{k}\right\} \subset\left(\Gamma_{1} \cup \cdots \cup \Gamma_{i}\right)$. This justifies the second line of the inner sum.

Finally, consider any moduli space which does not have positive punctures at orbits in the collections $\Gamma_{i_{1}}, \ldots, \Gamma_{i_{l}}$. Such moduli space appears $2^{l}$ times in the coefficient of $q^{\Gamma} q^{\left(\Gamma_{1} \cup \ldots \cup \Gamma_{i}\right) \backslash\left\{\gamma_{1}, \ldots, \gamma_{k}\right\}}$ in the expression $\mu_{i j}\left(q^{\Gamma_{1}}, \ldots, q^{\Gamma_{i}}\right)$. More specifically, for $i_{m}=i_{1}, \ldots, i_{l}$, it appears in both $\left(\cdots q^{\Gamma_{i_{m}}} \cdots H \cdots\right)$ and $\left(\cdots H \cdots q^{\Gamma_{i m}} \cdots\right)$ with opposite signs, since $H$ is odd degree. This justifies the middle sum and the top line of the inner sum.

One could alternatively define $\mu=\sum_{i j} \mu_{i j}$ using the above moduli spaces and prove that they satisfy the $L_{\infty}$ relations. As this is logically redundant, we omit this proof, but it is an interesting exercise in using a Gromov compactness argument.

Proposition 3.8. The quasi-isomorphism class of the quantum $L_{\infty}$ algebra of a contact manifold $M$ is a contact invariant.

Proof. As mentioned in Section 3.3, the authors in [3] re-interpret the SFT Hamiltonian $H$ in $[6]$ as a differential $D$, which is the same as the $\mu_{1}$ of the quantum $L_{\infty}$ algebra on $\mathcal{A}[[\hbar]]$. They point out how the potential $F$ associated to an exact symplectic cobordism between contact manifolds $M_{+}, M_{-}$ gives rise to a morphism

$$
\vec{F}: \mathcal{A}_{+} \rightarrow \mathcal{A}_{-}[[\hbar]], \quad e^{\vec{F}} D_{+}=D_{-} e^{\vec{F}} .
$$


Since $\mu_{k}$ is derived from multiple brackets with $\mu_{1}, e^{F}$ defines an $L_{\infty}$ morphism between the two quantum $L_{\infty}$ structures associated to $M_{-}$and $M_{+}$.

Remark 3.9. In general, if $(W, H, \mathcal{A}[[\hbar]], \varphi, \iota)$ and $\left(W^{\prime}, H^{\prime}, \mathcal{A}^{\prime}[[\hbar]], \varphi^{\prime}, \iota^{\prime}\right)$ are two polarized quantum backgrounds with $\mathcal{A}[[\hbar]]=W / I$ and $\mathcal{A}^{\prime}[[\hbar]]=W^{\prime} / I^{\prime}$, then an algebra map $\alpha: W \rightarrow W^{\prime}$ with $\alpha(I) \subset I^{\prime}$ and $\alpha(H)=H^{\prime}$ induces a map $\tau: \mathcal{A}[[\hbar]] \rightarrow \mathcal{A}^{\prime}[[\hbar]]$ and the pair $(\alpha, \tau)$ defines a morphism of backgrounds. So $\tau$ defines a morphism between the quantum $L_{\infty}$ algebras $\left(\mathcal{A}[[\hbar]],\left\{\mu_{i}\right\}\right)$ and $\left(\mathcal{A}^{\prime}[[\hbar]],\left\{\mu_{i}^{\prime}\right\}\right)$. Not all morphisms between these $L_{\infty}$ algebras are of this type: in general, an $L_{\infty}$ morphism would consist of maps $F_{1}, F_{2}, F_{3}, \ldots$ with $F_{i}: \mathcal{A}[[\hbar]]^{\otimes i} \rightarrow \mathcal{A}^{\prime}[[\hbar]]$, while those induced by polarized background morphisms only have the $F_{1}$ map. In particular, the morphisms in Proposition 3.8 between the quantum $L_{\infty}$ algebras associated to contact manifolds that arise from exact cobordisms only have an $F_{1}$ term.

At this point, we contrast the classical and quantum $L_{\infty}$ structures to the level zero $L_{\infty}$ algebra mentioned in Section 2. Recall the classical $L_{\infty}$ algebra $\left(\mathcal{A},\left\{\mu_{k 0}\right\}\right): \mu_{k 0}: \mathcal{A}^{\otimes k} \rightarrow \mathcal{A}$. In particular, $\mu_{10}: \mathcal{A} \rightarrow \mathcal{A}$ is a square zero, degree one operator, which by Proposition 3.7 is equivalent to the contact homology differential. As mentioned in Section 2, for any polarized quantum background, we can expand $d=\sum_{k=0} d_{k}$. If $d_{0}=0$, this is dual to the level zero $L_{\infty}$ algebra. (This can be relaxed with the use of augmentations, see Section 3.6.) Each moduli space of genus 0 rigid $J$-holomorphic curves contributes to just one of the operations in the level zero $L_{\infty}$ algebra. By contrast, in the quantum $L_{\infty}$, a given moduli space (of any genus) is counted for multiple operations. For example, $\mathcal{M}_{0}^{1}\left(\left\{p^{\gamma_{1}}, p^{\gamma_{2}}\right\}\right)$ contributes to both $\mu_{20}$ and $\mu_{11}$. To summarize,

\{quantum background $(\mathcal{W}, H, N, \varphi)\}$

$$
\begin{aligned}
& \text { if polarized } \underset{\rightsquigarrow}{\rightsquigarrow}\left\{\text { quantum } L_{\infty} \text { algebra }\left(\mathcal{A}[[\hbar]],\left\{\mu_{k}\right\}\right)\right\} \\
& \stackrel{\hbar=0}{\rightsquigarrow}\left\{\text { classical } L_{\infty} \text { algebra }\left(\mathcal{A},\left\{\mu_{k 0}\right\}\right)\right\} \\
&\text { if Weyl type and } \left.d_{0}=0 \text { the level zero } L_{\infty} \text { coalgebra }\left(V,\left\{d_{r}\right\}\right)\right\}
\end{aligned}
$$

Degree one elements $H$ in a Weyl algebra satisfying the two conditions are closely related to algebras over the cobar dual of the Frobenius co-properad [5]. These algebras include all of the operations that algebras over a resolution of the involutive bi-Lie properad would have, and possibly some other operations as well.

3.5. Quantum master equation. In this section we propose a way to process the quantum background coming from the SFT of a contact manifold as a first step to extracting invariants. We defined the algebraic structures 
on the module of a polarized background in the previous section to provide a familiar language for describing the process.

Definition 3.10. Let $B=(W, N, H, \varphi)$ be a background and let $A$ be a nilpotent, commutative $k[[\hbar]]$ algebra, and $\pi \in(W \otimes A)^{0}$. We call the equation

$$
\left(e^{\pi / \hbar} H e^{-\pi / \hbar}\right) \varphi=0
$$

the QME and say that $\pi$ is a solution to the QME.

One may linearize the QME in two ways, leading to two homology theories: one with $\hbar$ 's called the Dirac space, and one without $\hbar$ 's called the tangent space. In general, one defines these homology theories by equivalence classes of solutions to the QME with parameters in the rings $A=k[[t]] / t^{2}$ or $A=k[[t, \hbar]] / t^{2}$. In the physics language, the tangent space consists of equivalence classes of classical observables, and the Dirac space consists of equivalence classes of quantum observables.

For polarized quantum backgrounds, both the QME and the associated homology theories are easily expressed in terms that have already been defined. If the background $B$ is polarized, and $\pi \in \iota N \otimes A \subset W \otimes A$, then $\pi$ satisfies the QME if and only if $\pi$ satisfies the Maurer-Cartan equation

$$
\mu_{1}(\pi)+\frac{1}{2 !} \mu_{2}(\pi, \pi)+\frac{1}{3 !} \mu_{3}(\pi, \pi, \pi)+\cdots=0
$$

in the quantum $L_{\infty}$ algebra associated to $B$. This is seen by expanding the exponential $\left(e^{\pi / \hbar} H e^{-\pi / \hbar}\right)$ in terms of iterated brackets $1+\frac{1}{\hbar}[H, \pi]+$ $\frac{1}{2 ! \hbar^{2}}[[H, \pi], \pi]+\cdots$ We identify $\pi \in N$ with its image $\iota(\pi) \in W$ when it causes no confusion.

Definition 3.11. Let $B=(W, H, \mathcal{A}[[\hbar]], \varphi, \iota)$ be a polarized background. We define the Dirac space of $B$ to be the homology of the quantum $L_{\infty}$ algebra $\left(A[[h]], u_{k}\right)$. We define the tangent space of $B$ to be the homology of the classical $L_{\infty}$ algebra $\left(A, u_{k 0}\right)$.

Let $B=(W, H, N, \varphi, \iota)$ be a polarized quantum background and consider the ring $A=k\left[\left[\mathcal{H}^{*}, \hbar\right]\right]$, where $\mathcal{H}$ is the tangent space of $B$. A solution $\Pi \in W \widehat{\otimes} A$ to the QME is called versal if and only if it satisfies the condition that the linear terms span the tangent space. That is, if

$$
\Pi=\left(\gamma_{i}^{0}+\gamma_{i}^{1} \hbar+\gamma_{i}^{2} \hbar^{2}+\cdots\right) \otimes t^{i}+\left(\gamma_{i j}^{0}+\gamma_{i j}^{1} \hbar+\cdots\right) \otimes t^{i} t^{j}+\cdots
$$

satisfies the QME and $\left\{\left[\gamma_{i}^{0}\right]\right\}$ form a basis of $\mathcal{H}$ dual to $t^{i} \in \mathcal{H}^{*}$. Of course, the ring $k\left[\left[\mathcal{H}^{*}, \hbar\right]\right]$ is not nilpotent, but by "satisfies the QME", we mean "satisfies the QME to each finite order in $t$ ".

The picture to have in mind is that of a neighborhood of a point in the moduli space of solutions. The point corresponds to the 0 solution to the QME. The tangent space $\mathcal{H}$ is the tangent space to the moduli space at 
the point. A versal solution $\Pi$ gives a map from $\mathcal{H}$ to the moduli space by $t \mapsto \Pi(t)$, and gives coordinates on this neighborhood. At the point in the moduli space with coordinate $t$, one can define a background

$$
B_{t}:=\left(\mathcal{W}, e^{\Pi(t) / \hbar} e^{-\Pi(t) / \hbar}, N, \varphi, \iota\right),
$$

which is a background precisely because $\Pi$ satisfies the QME: $\left(e^{\Pi / \hbar} H e^{-\Pi / \hbar}\right)$ $\varphi=0$. Then one can view the tangent bundle of this neighborhood by identifying the tangent space at $t$ with the tangent space of $B_{t}$, the background represented by the point $t$.

Theorem 3.12 ([10]). For every versal solution to the QME, there exists a flat quantum super connection on the tangent bundle in a neighborhood of zero in the moduli space of backgrounds.

In special coordinates, this superconnection encodes the essential physical invariants of the theory associated to the background. The Frobenius manifold structure (minus the inner product) on a moduli space arising from the formality of Lie polyvector fields on a Calabi-Yau manifold [1] provides an example.

One can write down the condition for $\Pi$ to be a versal solution in powers of $t$ and $\hbar$ and imagine constructing a solution term by term, beginning with $\gamma_{i}^{0} t^{i}$, where $\left\{\left[\gamma_{i}^{0}\right]\right\}$ is a basis for $\mathcal{H}$. One obtains a collection of obstructions, indexed by all powers of $t$ (and all powers of $\hbar$ ), that must all vanish in order for $\Pi$ to exist. For example, in order to be able to extend the infinitesimal solution $\gamma_{i}^{0} t^{i}$ to a versal one, it is necessary that the linear in $t$ obstructions vanish. That is, for each $i$, there exist $\gamma_{i}^{j} \in \iota(V) j=1,2, \ldots$ so that for $\gamma:=\gamma_{i}^{0}+\hbar \gamma_{i}^{1}+\hbar^{2} \gamma_{i}^{2}+\cdots$ we have $\mu_{1}(\gamma)=0$. The surprising result is that this condition is also sufficient: if the linear in $t$ obstructions vanish, then all obstructions vanish.

Theorem 3.13. There exists a versal solution to the $Q M E$ in a polarized quantum background $(\mathcal{W}, H, N, \varphi, \iota)$ if and only if there exists a basis of representatives $\left\{\gamma_{i}^{0}\right\}$ of the tangent space that can be extended to representatives $\gamma_{i}=\gamma_{i}^{0}+\hbar \gamma_{i}^{1}+\hbar^{2} \gamma_{i}^{2}+\cdots$ of the Dirac space.

Proof. The same proof for the case of differential BV algebras $[\mathbf{9}]$ works for the general case of quantum backgrounds. In fact, Lemma 2 in $[\mathbf{9}]$ is simply a re-expression of the $\mathrm{QME}$ for differential BV algebras in the quantum background form, and then a quantum background proof is given.

We translate Theorem 3.13 to the language of $J$-holomorphic curves in the SFT case. There exists a versal solution if and only if there is a basis for the contact homology, $\left\{q^{\Gamma_{i}^{0}}\right\}_{i}$, so that each $q^{\Gamma_{i}^{0}}$ can be extended to

$$
q^{\Gamma_{i}}=\sum_{j=0} q^{\Gamma_{j}^{i} \hbar^{j}}
$$


satisfying the following: for all $n \geq 1$ and all $q^{\Gamma} \in \mathcal{A}$,

$$
\sum_{j=0}^{n} \sum_{k=1}^{1+j} \sum_{\left\{\gamma_{1}, \ldots, \gamma_{k}\right\} \subset \Gamma_{n-j}^{i}} \#\left(\mathcal{M}_{1+j-k}^{1}\left(\left\{\gamma_{1}, \ldots, \gamma_{k}\right\}, \Gamma\right)=0 .\right.
$$

So, there is a two-tiered approach to understanding invariants of a polarized quantum background. First, try to extend the representatives of the tangent space to representatives of the Dirac space. If this is possible, then there exists a universal solution $\Pi$ to the QME, and one can construct it algorithmically from the tangent-to-Dirac extension. From the versal solution, one can construct the flat quantum superconnection. If one encounters obstructions to extending the tangent space to the Dirac space, then those obstructions are invariants of the background which can be organized systematically. The obstructions to extending the tangent space to the Dirac space satisfy their own set of internal relations, which are organized as a certain differential. We describe this in Theorem 3.14.

Let us analyze the obstructions to the existence of the extension

$$
\gamma_{i}^{0} \mapsto \gamma_{i}^{0}+\hbar \gamma_{i}^{1}+\cdots,
$$

where $\left\{\left[\gamma_{i}^{0}\right]\right\}$ is a basis for the $\mu_{10}$ homology and $\gamma_{i}^{0}+\hbar \gamma_{i}^{1}+\cdots$ is $\mu_{1}$-closed. The first obstruction is

$$
\mu_{11} \gamma_{i}^{0}=\mu_{10} \gamma_{i}^{1}
$$

For the extension to exist, the expression $\mu_{11} \gamma_{i}^{0}$ must be $\mu_{10}$-exact. In general, $\mu_{11} \gamma_{i}^{0}$ will be $\mu_{10}$-closed, but not necessarily exact. However, having chosen representatives $\left\{\gamma_{i}^{0}\right\}$ gives a way to write it as closed plus exact. The closed part defines the first part of a differential $\kappa$ and the exact part defines the first part of a map $\beta$ from $\mathcal{H} \rightarrow \mathcal{A}[[\hbar]]$.

Theorem 3.14. Let $B=(\mathcal{W}, H, \mathcal{A}[[\hbar]], \varphi)$ be a polarized quantum background. For every basis $\left\{\gamma_{i}^{0}\right\}$ of representatives of the tangent space, there is map of $k[[\hbar]]$ modules $\kappa: \mathcal{H}[[\hbar]] \rightarrow \mathcal{H}[[\hbar]]$ satisfying $\kappa^{2}=0$ and $\kappa(\mathcal{H}) \subset \hbar \mathcal{H}[[\hbar]]$, and a map of complexes $\beta:(\mathcal{H}[[\hbar]], \kappa) \rightarrow\left(\mathcal{A}[[\hbar]], \mu_{1}\right)$ of the form $\left[\gamma_{i}^{0}\right] \mapsto \gamma_{i}^{0}+\hbar \gamma_{i}^{1}+\hbar^{2} \gamma_{i}^{2}+\cdots$

Proof. One can give an abstract argument by noting that $(\mathcal{H}, 0)$ and $\left(\mathcal{A}, \mu_{10}\right)$ are quasi-isomorphic as differential graded vector spaces and that $\mu_{10}$ can be deformed over $k[[\hbar]]$ to

$$
\mu_{1}=\mu_{10}+\mu_{11} \hbar+\mu_{12} \hbar^{2}+\cdots,
$$

and therefore, a general deformation theory argument implies that the differential 0 can also be extended to

$$
\kappa=0+\kappa_{1} \hbar+\kappa_{2} \hbar^{2}+\cdots
$$

and that the quasi-isomorphism between $(\mathcal{H}, 0)$ and $\left(\mathcal{A}, \mu_{10}\right)$ can be extended over $k[[\hbar]]$ to the map $\beta$. However, it might be instructive to illustrate a 
construction of $\kappa$ and $\beta$ so that one might carry it out in the SFT example. Begin with a basis $\left\{\gamma_{i}^{0}\right\}$ of representatives for the tangent space $H\left(\mathcal{A}, \mu_{10}\right)$. We define the map $\beta$ modulo $\hbar$ by

$$
\left[\gamma_{i}^{0}\right] \stackrel{\beta}{\mapsto} \gamma_{i}^{0} .
$$

Now, since $\mu_{10}\left(\gamma_{i}^{0}\right)=0$,

$$
\mu_{1}\left(\gamma_{i}^{0}\right)=\hbar \mu_{11}\left(\gamma_{i}^{0}\right)+\hbar^{2} \mu_{12}\left(\gamma_{i}^{0}\right)+\cdots
$$

and $\mu_{1}^{2}=0$ implies that

$$
0=\hbar\left(\mu_{10} \mu_{11} \gamma_{i}^{0}\right)+\hbar^{2}\left(\mu_{11}^{2} \gamma_{i}^{0}+\mu_{10} \mu_{12} \gamma_{i}^{0}\right)+\hbar^{3} \cdots
$$

From the $\hbar^{1}$ part of equation (3.3), we see that $\mu_{11} \gamma_{i}^{0}$ is $\mu_{10}$-closed. Therefore, there exist constants $\kappa_{1 i}^{j}$ and elements $\gamma_{i}^{1} \in \mathcal{A}$ so that

$$
\mu_{11} \gamma_{i}^{0}=\kappa_{1 i}^{j} \gamma_{j}^{0}+\mu_{10} \gamma_{i}^{1} .
$$

We now extend the definitions of $\beta$ and $\kappa$ modulo $\hbar^{2}$ to be

$$
\begin{gathered}
{\left[\gamma_{i}^{0}\right] \stackrel{\beta}{\mapsto} \gamma_{i}^{0}+\hbar \gamma_{i}^{1},} \\
\kappa\left(\left[\gamma_{i}^{0}\right]\right)=\hbar \kappa_{1 i}^{j}\left[\gamma_{j}^{0}\right] .
\end{gathered}
$$

Now, substituting the RHS of equation (3.4) in for $\mu_{11} \gamma_{i}^{0}$ in the $\hbar^{2}$ part of equation (3.3) gives

$$
\mu_{11}\left(\kappa_{1 i}^{j} \gamma_{j}^{0}+\mu_{10} \gamma_{i}^{1}\right)+\mu_{10} \mu_{12} \gamma_{i}^{0}=0 .
$$

Substituting the RHS of equation (3.4) in for $\mu_{11} \gamma_{i}^{0}$ again (and using the fact that $\mu_{10}$ and $\mu_{11}$ commute, which follows from $\mu_{1}^{2}=0$ ) produces

$$
\kappa_{1 i}^{j} \kappa_{1 j}^{k} \gamma_{k}^{0}+\mu_{10}\left(\kappa_{1 i}^{j} \gamma_{j}^{1}+\mu_{11} \gamma_{i}^{1}+\mu_{12} \gamma_{i}^{0}\right)=0 .
$$

Since $\left\{\gamma_{k}^{0}\right\}$ are a basis of $H\left(\mathcal{A}, \mu_{10}\right)$, equation (3.5) implies that both terms on the LHS vanish separately:

$$
\kappa_{1 i}^{j} \kappa_{1 j}^{k}=0
$$

and

$$
\mu_{10}\left(\kappa_{1 i}^{j} \gamma_{j}^{1}+\mu_{11} \gamma_{i}^{1}+\mu_{12} \gamma_{i}^{0}\right)=0 .
$$

So we see $\kappa_{1}^{2}=0$, and as they are defined thus far, we have

$$
\beta \kappa_{1}\left[\gamma_{i}^{0}\right]=\beta \hbar \kappa_{1 i}^{j}\left[\gamma_{i}^{0}\right]=\hbar \kappa_{1 i}^{j} \gamma_{i}^{0}+\hbar^{2} \kappa_{1 i}^{j} \gamma_{i}^{1}
$$

and

$$
\mu_{1} \beta\left[\gamma_{i}^{0}\right]=\mu_{1}\left(\gamma_{i}^{0}+\hbar \gamma_{i}^{1}\right)=\hbar\left(\mu_{11} \gamma_{i}^{0}+\mu_{10} \gamma_{i}^{1}\right)+\hbar^{2}\left(\mu_{12} \gamma_{i}^{0}+\mu_{11} \gamma_{i}^{1}\right)+\hbar^{3} \cdots
$$


which shows that $\beta$ is a chain map modulo $\hbar^{2}$. For the $\hbar^{2}$ step, note that equation (3.7) implies that there exist constants $\kappa_{2 i}^{j}$ and $\gamma_{i}^{2} \in \mathcal{A}$ so that

$$
\kappa_{1 i}^{j} \gamma_{j}^{1}+\mu_{11} \gamma_{i}^{1}+\mu_{12} \gamma_{i}^{0}=\kappa_{2 i}^{j} \gamma_{i}^{0}+\mu_{10} \gamma_{i}^{2} .
$$

Now extend the definitions of $\beta$ and $\kappa$ modulo $\hbar^{3}$ to be

$$
\begin{gathered}
{\left[\gamma_{i}^{0}\right] \stackrel{\beta}{\mapsto} \gamma_{i}^{0}+\hbar \gamma_{i}^{1}+\hbar^{2} \gamma_{i}^{2},} \\
\kappa\left(\left[\gamma_{i}^{0}\right]\right)=\left(\hbar \kappa_{1 i}^{j}+\hbar^{2} \kappa_{2 i}^{j}\right)\left[\gamma_{j}^{0}\right] .
\end{gathered}
$$

And so on.

Like Massey products, $\kappa$ is constructed with choices. Write $\kappa=\hbar \kappa_{1}+$ $\hbar^{2} \kappa_{2}+\cdots$, where each $\kappa_{i}: \mathcal{H} \rightarrow \mathcal{H}$. The first map $\kappa_{1}$ is just the image of $\mu_{11}$ in $\mu_{10}$ homology, which is well defined by (3.3). The second map $\kappa_{2}$ depends on the choice of $\gamma_{i}^{1}$ in equation (3.4), specifically on $\kappa_{1}\left(\gamma_{i}^{1}\right)$. Therefore, if $\kappa_{1}$ vanishes, $\kappa_{2}$ is defined independently of any choices. And so on, if $\kappa_{i}=0$ for $i=1, \ldots, j-1$, then $\kappa_{j}$ is defined independently of any choices.

The existence of a versal solution to the QME is a quasi-isomorphism invariant of the $L_{\infty}$ algebra. It follows that whether there exists a basis $\left\{\gamma_{i}^{0}\right\}$ of the contact homology $\mathcal{H}=H\left(\mathcal{A}, d=\mu_{10}\right)$ and a choice of $\kappa=0$ is a contact invariant: if $\kappa=0$, there is an extension

$$
\gamma_{i}^{0} \mapsto \beta\left(\left[\gamma_{i}^{0}\right]\right)=\gamma_{i}^{0}+\hbar \gamma_{i}^{1}+\cdots
$$

of the tangent space $\mathcal{H}$ to the Dirac space. However, the vanishing of $\kappa$ is independent of any choices, including the choice of basis. Therefore, it makes sense to speak of whether or not $\kappa$ vanishes, without reference to any bases or choices, and that the vanishing or non-vanishing of $\kappa$ is a contact invariant.

Definition 3.15. We call the map $\kappa$ in Theorem 3.14 the kappa invariant.

There exists a versal solution to the QME if and only if $\kappa=0$. If $\kappa \neq 0$, then the components of $\kappa$, defined modulo some choices, define maps $\kappa_{i}$ : $\mathcal{H} \rightarrow \mathcal{H}$ which encode the obstructions to finding a versal solution to the QME. In the SFT case, these obstructions have higher genus information, but are not independent - they satisfy relations among themselves in the equation $\kappa^{2}=\left(\hbar \kappa_{1}+\hbar^{2} \kappa_{2}+\cdots\right)^{2}=0$. The idea of using higher genus holomorphic curves to define structures on a homology theory that is defined using no genus also appears in [6], where the full theory of curves in $H$ is used to define a "Satellite" structure on the homology of their graded Poisson algebra (which is a restricted theory of curves with no genus). It would be interesting to compare these two reductions. 
3.6. Augmentations for polarized quantum backgrounds. A directed symplectic cobordism $C$ from the empty contact manifold to $M$ is called a symplectic filling of $M$. Let $\pi$ denote the potential associated to $C$. Since one boundary of the cobordism is empty, there are no $q^{-}$variables and the potential $\pi \in \mathcal{W}(M)$. The standard SFT results on augmentations (see, for example, $[\mathbf{3}]$ ) can be re-expressed in the language of quantum backgrounds to say that $\pi$ is a solution to the QME. Furthermore, the polarized background

$$
\left(\mathcal{W}(M), e^{\pi / \hbar} H e^{-\pi / \hbar}, N, 1, \iota\right)
$$

has "no $d_{0}$ " and so the level zero $L_{\infty}$ structure is defined.

In the general picture where the background associated to the SFT of a contact manifold $M$ represents a zero point in the moduli space of solutions to the QME, one has a fibration whose fibers are quantum $L_{\infty}$ structures. One may set $\hbar=0$ and obtain a family of classical $L_{\infty}$ structures, fibered over the moduli space. For each point $t$ in the moduli space, one has $\mu_{1}(t)$, which may be a weak $L_{\infty}$ structure, or if $d_{0}(t)=0$, it may be a $L_{\infty}$ structure. From the perspective of quantum backgrounds, the important thing is to understand how the family of quantum $L_{\infty}$ structures varies with the moduli. It is this variation of structure (at a smooth point) that is encapsulated by the flat superconnection of Theorem 3.12, and it is the way in which this variation fails to vary smoothly (at a nonsmooth point) that is encapsulated by the kappa invariant. By focussing on the points where the classical $L_{\infty}$ structure is linearizable, one loses the possibility to study how these structures vary with the moduli since these points are likely to be isolated. However, if one is most interested in cylindrical-type contact homology theories, one can certainly look at the full set of them, which potentially carries more geometric information than a single one.

\section{The unit cotangent bundle example}

We conclude this paper with a computation.

4.1. String topology and geodesics. Consider an $n$-dimensional manifold $X$. Let $\Omega^{\mathrm{eq}}(X)=\Omega(X) \times_{S^{1}} E S^{1}$ denote its equivariant loop space, and let $K$ denote the constant loops. Chas and Sullivan $[\mathbf{2}]$ define the degree $(2-n)$ bracket and cobracket string topology operations

$$
\begin{gathered}
{[\cdot, \cdot]: H_{j}\left(\Omega^{e q}(X), K\right) \otimes H_{i}\left(\Omega^{\mathrm{eq}}(X), K\right) \rightarrow H_{i+j-n+2}\left(\Omega^{\mathrm{eq}}(X), K\right),} \\
\Delta: H_{j}\left(\Omega^{\mathrm{eq}}(X), K\right) \rightarrow \oplus_{i} H_{j-n+2-i}\left(\Omega^{\mathrm{eq}}(X), K\right) \otimes H_{i}\left(\Omega^{\mathrm{eq}}(X), K\right)
\end{gathered}
$$

motivated by the Goldman bracket and Turaev cobrackets on surfaces.

Given a basis $\lambda_{i}$ of $H_{*}\left(\Omega^{\mathrm{eq}}(X), K\right)$, define $\left\langle\Delta \lambda_{i}, \lambda_{j} \lambda_{k}\right\rangle$ by

$$
\Delta \lambda_{i}=\sum_{k, l}\left\langle\Delta \lambda_{i}, \lambda_{j} \lambda_{k}\right\rangle \lambda_{j} \lambda_{k} .
$$


Define $\left\langle\left[\lambda_{i}, \lambda_{j}\right], \lambda_{k}\right\rangle$ similarly.

These operations are moreover defined not just at the homology level, but also on certain chains of the equivariant loop space. There are several different attempts to resolve certain transversality issues thereby defining these operations on all chains $[\mathbf{4}, \mathbf{1 1}]$.

If $X$ is a Riemannian manifold with a generic metric, then its closed geodesics generate a Morse complex, graded by the Morse index, with the differential given by gradient flows of the $L^{2}$-energy functional. The bracket and cobracket are not yet rigorously defined on this Morse complex unless the differential vanishes. If $X$ is hyperbolic, the differential vanishes; however, if $\operatorname{dim}(X)>2$, the string topology operations vanish. The case $\operatorname{dim}(X)=2$ is considered in Section 4.2.

The metric on $X$ also induces a canonical contact one-form for the unit cotangent bundle $(M, \xi)=\left(S T^{*} X, \operatorname{ker}\left(\sum x_{i} d y_{i}\right)\right)$, where $x_{i}$ is a local coordinate system for $X$ and $y_{i}$ is a cotangent vector dual to $\partial_{x_{i}}$. Moreover, the oriented closed geodesics of $X$ are in one-to-one correspondence with the Reeb orbits of $M, \lambda \leftrightarrow \gamma_{\lambda}$, and the Conley-Zehnder index $\mathrm{CZ}\left(\gamma_{\lambda}\right)$ equals the Morse index $m(\lambda)$.

4.2. Riemann surface of genus at least 2 . Let $X$ be a Riemann surface of genus at least 2, so that it admits a metric on whose oriented geodesics, bracket and cobracket operations are well defined and non-trivial. To simplify notation while applying the above correspondence between geodesics $\lambda$ and Reeb orbits $\gamma_{\lambda}$, denote the generator $p^{\gamma_{\lambda}}$ in $\mathcal{W}\left(S T^{*} X\right)$ by $p^{\lambda}$. Similarly, denote $q^{\gamma_{\lambda}}$ by $q^{\lambda}$.

One computes

$$
\operatorname{dim} \mathcal{M}_{g}\left(\left\{\gamma_{\lambda_{1}}, \ldots, \gamma_{\lambda_{k}}\right\},\left\{\gamma_{\eta_{1}}, \ldots, \gamma_{\eta_{l}}\right\}\right)=-2+2 g+k+l
$$

Since $H$ counts those holomorphic curves which appear in one-dimensional moduli spaces, this computation implies that $H$ can only have non-zero coefficients in front of terms of the form

$$
p p^{\prime} p^{\prime \prime} \hbar^{-1}, \quad p p^{\prime} q^{\prime \prime} \hbar^{-1}, \quad p q^{\prime} q^{\prime \prime} \hbar^{-1}, \quad p \hbar^{0}
$$

where a priori, $p, p^{\prime}, p^{\prime \prime}, q^{\prime}, q^{\prime \prime}$ can be any of the Weyl algebra generators.

Note that the disk cotangent bundle is a symplectic filing of the unit cotangent bundle $M$. By observing what curves appeared in this filing of $M$, Cieliebak and Latschev [3] could (modulo analysis) connect $H$ to the string topology operations on the surface $X$. Let $\bar{\lambda}_{1}$ be the geodesic $\lambda_{1}$ with opposite orientation. Like the bracket and cobracket, this is a degree 0 operation.

Let $Z_{n} \subset S_{n}$ denote the subgroup of cyclic permutations. 
Proposition $4.1([3])$. The $S F T$ of $S T^{*} X$ is given by, up to signs,

$$
\begin{aligned}
H= & \frac{1}{\hbar} \sum_{\lambda_{1}, \lambda_{2}, \lambda_{3}}\left[\left\langle\Delta \lambda_{1}, \lambda_{2} \lambda_{3}\right\rangle p^{\lambda_{1}} q^{\lambda_{2}} q^{\lambda_{3}}\right. \\
& +\left(\left\langle\left[\lambda_{1}, \lambda_{2}\right], \lambda_{3}\right\rangle+\sum_{\tau \in Z_{2}}\left\langle\Delta \lambda_{\tau(1)}, \bar{\lambda}_{\tau(2)} \lambda_{3}\right\rangle\right) p^{\lambda_{1}} p^{\lambda_{2}} q^{\lambda_{3}} \\
& \left.+\left(\sum_{\tau \in Z_{3}}\left(\left\langle\left[\lambda_{\tau(1)}, \lambda_{\tau(2)}\right], \bar{\lambda}_{\tau(3)}\right\rangle+\left\langle\Delta \lambda_{\tau(1)}, \bar{\lambda}_{\tau(2)} \bar{\lambda}_{\tau(3)}\right\rangle\right)\right) p^{\lambda_{1}} p^{\lambda_{2}} p^{\lambda_{3}}\right] \\
& +\frac{1}{2} \sum_{\lambda_{1}, \lambda_{2}}\left\langle\Delta \lambda_{1}, \lambda_{2} \bar{\lambda}_{2}\right\rangle p^{\lambda_{1}} \hbar^{0} .
\end{aligned}
$$

The factorial coefficients appearing in the definition of $H$ in Section 2 are canceled by the "over-counting" from permuting the ordering of the marked domain points.

4.3. The general case. We now generalize Cieliebak and Latschev's example. We remark that this generalization does not require any analytical results for $J$-holomorphic curves. Consider a closed $n$-dimensional Riemann manifold $X$ where the count of $J$-holomorphic curves to determine $H \in \mathcal{W}\left(S T^{*} X\right)$ is unknown.

We would like to give $X$ the same graded Weyl algebra, $\mathcal{W}\left(S T^{*} X\right)$, as before; that is, the one generated by the oriented geodesics. However, unlike in Section 4.2, the relevant string topology operations are not yet rigorously defined; thus, we instead let $\mathcal{W}(X)$ denote the Weyl algebra generated by a fixed set of generators for $H_{*}\left(\Omega^{\mathrm{eq}}(X), K\right)$, homogeneous in degree.

For such $\lambda \in H_{i}\left(\Omega^{\mathrm{eq}}(X), K\right)$, we have the degree 0 operation induced by orientation reversing, $\bar{\lambda}$, which is defined in a way such that its square is given by $\overline{\bar{\lambda}}=(-1)^{n+1} \lambda$. We also have the degree $(2-n)$ bracket and cobracket operations. For each $\lambda$, let $p^{\lambda}, q^{\lambda}$ denote generators of $\mathcal{W}(X)$ with gradings

$$
\left|p^{\lambda}\right|=-i+n-3, \quad\left|q^{\lambda}\right|=i+n-3 .
$$

For generators $\lambda_{1} \in H_{i_{1}}\left(\Omega^{\mathrm{eq}}(X), K\right), \quad \lambda_{2} \in H_{i_{1}}\left(\Omega^{\mathrm{eq}}(X), K\right), \quad \lambda_{3} \in$ $H_{i_{3}}\left(\Omega^{\mathrm{eq}}(X), K\right)$ we can consider coefficients

$$
\left\langle\Delta \lambda_{1}, \lambda_{2} \lambda_{3}\right\rangle \text {, which is non-zero only if } i_{1}-i_{2}-i_{3}=n-2
$$

and

$$
\left\langle\left[\lambda_{1}, \lambda_{2}\right], \lambda_{3}\right\rangle, \quad \text { which is non-zero only if } i_{1}+i_{2}-i_{3}=n-2 .
$$

With this notation in mind, we can now make sense of the formula in Proposition 4.1 for any manifold $X$. Label this element as $H^{\prime} \in \mathcal{W}(X)$, since it may not agree with the holomorphically defined $H \in \mathcal{W}\left(S T^{*}(X)\right)$. 
Note that to have grading -1 (as should all terms in $H^{\prime}$ ), the signed sum of the homology gradings $i$ must be $n-2$. For example,

$$
-1=\left|p^{\lambda_{1}} p^{\lambda_{2}} q^{\lambda_{3}} \hbar^{-1}\right|=-i_{1}-i_{2}+i_{3}+n-3 \quad \rightarrow \quad i_{1}+i_{2}-i_{3}=n-2 .
$$

For certain terms, there are no obstructions due to grading. For example, in the expression $\left\langle\Delta \lambda_{1}, \lambda_{2} \lambda_{3}\right\rangle p^{\lambda_{1}} q^{\lambda_{2}} q^{\lambda_{3}} \hbar^{-1}$, we know that $i_{1}-i_{2}-i_{3}=n-2$ because the degree of $p^{\lambda_{1}} q^{\lambda_{2}} q^{\lambda_{3}} \hbar^{-1}$ is -1 . Thus, a priori, the count may be non-zero. However, for the expression $\left\langle\Delta \lambda_{1}, \bar{\lambda}_{2} \lambda_{3}\right\rangle p^{\lambda_{1}} p^{\lambda_{2}} q^{\lambda_{3}} \hbar^{-1}$ to be non-zero, grading considerations eliminate all those triples for which $i_{2} \neq 0$.

Proposition 4.2. The element $H^{\prime} \in \mathcal{W}(X)$ defined by the formula in Proposition 4.1 (without the overall factor of $\frac{1}{\hbar}$ ) is a structure element. In particular, the involutive Lie bi-algebra structure on the homology of the equivariant loop space can be re-interpreted as a quantum background.

Proof. There are six terms in $H^{\prime 2}$ which all must vanish. We see this is the case by re-interpreting each as a set of string topology relations. We use a slightly modified Einstein summation convention, where if the generator $x$ appears twice (or along with $\bar{x}$ ) in an expression (or product of expressions), then we sum over $x \in H_{*}\left(\Omega^{\mathrm{eq}}(X), K\right)$. Let $Z_{x_{1}, \ldots, x_{n}}$ denote the subgroup of cyclic permutations $Z_{n} \subset S_{n}$ which permutes the variables $x_{1}, \ldots, x_{n}$.

- The coefficients in front of terms of the form $p^{\lambda_{1}} q^{\lambda_{2}} q^{\lambda_{3}} q^{\lambda_{4}} \hbar^{1}$ are given by

$$
\sum_{Z_{\lambda_{2}, \lambda_{3}, \lambda_{4}}}\left\langle\Delta \lambda_{1}, x \lambda_{2}\right\rangle\left\langle\Delta x, \lambda_{3} \lambda_{4}\right\rangle=\left\langle\Delta^{2}\left(\lambda_{1}\right), \lambda_{2} \lambda_{3} \lambda_{4}\right\rangle
$$

which vanishes because $\Delta^{2}=0$; that is, the cobracket satisfies the co-Jacobi identity.

- The coefficients in front of terms of the form $p^{\lambda_{1}} p^{\lambda_{2}} q^{\lambda_{3}} q^{\lambda_{4}} \hbar^{1}$ are given by

$$
\begin{aligned}
& \left(\left\langle\left[\lambda_{1}, \lambda_{2}\right], x\right\rangle+\sum_{A_{\lambda_{1}, \lambda_{2}}}\left\langle\Delta \lambda_{1}, \bar{\lambda}_{2} x\right\rangle\right) \cdot\left\langle\Delta x, \lambda_{3} \lambda_{4}\right\rangle \\
& \quad+\sum_{Z_{\lambda_{1}, \lambda_{2}}, Z_{\lambda_{3}, \lambda_{4}}}\left\langle\Delta \lambda_{1}, \lambda_{3} x\right\rangle \cdot\left(\left\langle\left[x, \lambda_{2}\right], \lambda_{4}\right\rangle+\left\langle\Delta \lambda_{2}, \bar{x} \lambda_{4}\right\rangle+\left\langle\Delta x, \bar{\lambda}_{2} \lambda_{4}\right\rangle\right) .
\end{aligned}
$$

Here and throughout the proof, we will order these terms as they naturally appear after expanding the products. The third expression (which is in fact four terms) and the first expression sum to zero because of the compatibility of the bracket and cobracket

$$
\left\langle\Delta\left[\lambda_{1}, \lambda_{2}\right], \lambda_{3} \lambda_{4}\right\rangle=\left\langle\left[\Delta \lambda_{1}, \lambda_{2}\right], \lambda_{3} \lambda_{4}\right\rangle+\left\langle\left[\lambda_{1}, \Delta \lambda_{2}\right], \lambda_{3} \lambda_{4}\right\rangle
$$


$\Delta^{2}=0$ implies the second and the fifth expression individually are zero. The four terms in the fourth expression are

$$
\begin{aligned}
& \left\langle\Delta \lambda_{1}, \lambda_{3} x\right\rangle\left\langle\Delta \lambda_{2}, \lambda_{4} \bar{x}\right\rangle-\left\langle\Delta \lambda_{1}, \lambda_{3} x\right\rangle\left\langle\Delta \lambda_{2}, \lambda_{4} \bar{x}\right\rangle=0 . \\
& \left\langle\Delta \lambda_{1}, \lambda_{4} x\right\rangle\left\langle\Delta \lambda_{2}, \lambda_{3} \bar{x}\right\rangle-\left\langle\Delta \lambda_{1}, \lambda_{4} x\right\rangle\left\langle\Delta \lambda_{2}, \lambda_{3} \bar{x}\right\rangle=0 .
\end{aligned}
$$

Let us be more explicit in showing why the first of these two equations vanishes. (The second equation is similar.) Assume that $\alpha, \beta, \gamma, \delta$ and $\epsilon$ are generators of $H_{*}\left(\Omega^{\mathrm{eq}}(X), K\right)$, which for simplicity are assumed to be pairwise different, such that

$$
\langle\Delta \alpha, \beta \gamma\rangle \neq 0 \quad \text { and } \quad\langle\Delta \delta, \bar{\beta} \epsilon\rangle \neq 0 .
$$

Notice that the first equality used in the first line of $H^{\prime}$ gives a term $\langle\Delta \alpha, \beta \gamma\rangle p^{\alpha} q^{\beta} q^{\gamma} \hbar^{0}$, whereas the second equality used in the second line of $H^{\prime}$ gives a term $\langle\Delta \delta, \bar{\beta} \epsilon\rangle p^{\delta} p^{\beta} q^{\epsilon} \hbar^{0}$. Thus, in $H^{\prime 2}$, we obtain the following commutator with coefficient $\langle\Delta \alpha, \beta \gamma\rangle \cdot\langle\Delta \delta, \bar{\beta} \epsilon\rangle$ :

$$
\left[p^{\alpha} q^{\beta} q^{\gamma}, p^{\delta} p^{\beta} q^{\epsilon}\right] \hbar^{0}=(-1)^{s} p^{\alpha} p^{\delta}\left[q^{\beta}, p^{\beta}\right] q^{\gamma} q^{\epsilon} \hbar^{0} .
$$

Here $s$ is the sign obtained by the derivation rule of $[-,-]$ using the degrees of the $p$ s and $q \mathrm{~s}$.

On the other hand, using the first equality in the second line of $H^{\prime}$, we obtain a term $(-1)^{n+1}\langle\Delta \alpha, \beta \gamma\rangle p^{\alpha} p^{\bar{\beta}} q^{\gamma} \hbar^{0}$, and using the second equality in the first line of $H^{\prime}$, we obtain the term $\langle\Delta \delta, \bar{\beta} \epsilon\rangle p^{\delta} q^{\bar{\beta}} q^{\epsilon} \hbar^{0}$. In $H^{\prime 2}$, we get the following commutator with the same coefficient as above:

$$
(-1)^{n+1}\left[p^{\alpha} p^{\bar{\beta}} q^{\gamma}, p^{\delta} q^{\bar{\beta}} q^{\epsilon}\right] \hbar^{0}=(-1)^{s+n+1} p^{\alpha} p^{\delta}\left[p^{\bar{\beta}}, q^{\bar{\beta}}\right] q^{\gamma} q^{\epsilon} \hbar^{0} .
$$

Thus, we obtain opposite signs, due to the fact that $\left[q^{\beta}, p^{\beta}\right]=$ $-(-1)^{n-3}\left[p^{\beta}, q^{\beta}\right]=-(-1)^{n+1}\left[p^{\bar{\beta}}, q^{\bar{\beta}}\right]$, since $i_{\beta}=i_{\bar{\beta}}=0$.

- The coefficients in front of terms of the form $p^{\lambda_{1}} p^{\lambda_{2}} p^{\lambda_{3}} q^{\lambda_{4}} \hbar^{1}$ are given by

$$
\begin{aligned}
& \sum_{Z_{\lambda_{1}, \lambda_{2}, \lambda_{3}}}\left(\sum_{Z_{\lambda_{1}, \lambda_{2}}}\left\langle\Delta \lambda_{1}, \bar{\lambda}_{2} x\right\rangle+\left\langle\left[\lambda_{1}, \lambda_{2}\right], x\right\rangle\right) \\
& \cdot\left(\left\langle\Delta \lambda_{3}, \bar{x} \lambda_{4}\right\rangle+\left\langle\Delta x, \bar{\lambda}_{3} \lambda_{4}\right\rangle+\left\langle\left[x, \lambda_{3}\right], \lambda_{4}\right\rangle\right) \\
& +\sum_{Z_{\lambda_{1}, \lambda_{2}, \lambda_{3}}}\left\langle\Delta \lambda_{1}, \lambda_{4} x\right\rangle \cdot\left(\left\langle\Delta x, \bar{\lambda}_{2} \bar{\lambda}_{3}\right\rangle+\left\langle\left[\lambda_{2}, \lambda_{3}\right], \bar{x}\right\rangle\right. \\
& \left.+\sum_{Z_{\lambda_{2}, \lambda_{3}}}\left(\left\langle\Delta \lambda_{2}, \bar{x} \bar{\lambda}_{3}\right\rangle+\left\langle\left[x, \lambda_{2}\right], \bar{\lambda}_{3}\right\rangle\right)\right) .
\end{aligned}
$$


Expanding this out gives $3+3+4=10$ expressions. The sum of the first and ninth expressions, each made of six terms, vanishes due to pairwise cancelations:

$\sum_{Z_{\lambda_{1}, \lambda_{2}, \lambda_{3}}} \sum_{Z_{\lambda_{2}, \lambda_{3}}}\left(\left\langle\Delta \lambda_{1}, x \lambda_{4}\right\rangle \cdot\left\langle\Delta \lambda_{2}, \bar{x} \bar{\lambda}_{3}\right\rangle-\left\langle\Delta \lambda_{1}, x \lambda_{4}\right\rangle \cdot\left\langle\Delta \lambda_{2}, \bar{x} \bar{\lambda}_{3}\right\rangle\right)=0$.

The sign works just as in the previous case, namely non-vanishing coefficients $\langle\Delta \alpha, \beta \gamma\rangle$ and $\langle\Delta \delta, \bar{\epsilon} \bar{\beta}\rangle$ applied in the first and third lines of $H$ induce a commutator $\left[p^{\alpha} q^{\beta} q^{\gamma}, p^{\delta} p^{\epsilon} p^{\beta}\right] \hbar^{0}$, whereas applied twice in the second line induce the commutator $(-1)^{n+1}\left[p^{\alpha} p^{\bar{\beta}} q^{\gamma}, p^{\delta} p^{\epsilon} q^{\bar{\beta}}\right] \hbar^{0}$. We refer to this phenomenon as symmetry.

The sum of the second and the seventh vanishes due to co-Jacobi:

$$
\sum_{Z_{\lambda_{1}, \lambda_{2}, \lambda_{3}}}\left\langle\Delta^{2} \lambda_{1}, \bar{\lambda}_{2} \bar{\lambda}_{3} \lambda_{4}\right\rangle=0
$$

The sum of the third, fifth, and tenth vanishes due to the compatibility:

$\sum_{Z_{\lambda_{1}, \lambda_{2}, \lambda_{3}}}\left(\left\langle\Delta\left[\lambda_{1}, \lambda_{2}\right], \bar{\lambda}_{3} \lambda_{4}\right\rangle-\left\langle\left[\Delta \lambda_{1}, \lambda_{2}\right], \bar{\lambda}_{3} \lambda_{4}\right\rangle-\left\langle\left[\lambda_{1}, \Delta \lambda_{2}\right], \bar{\lambda}_{3} \lambda_{4}\right\rangle\right)=0$.

The sum of the fourth and eighth vanishes due to symmetry:

$$
\sum_{Z_{\lambda_{1}, \lambda_{2}, \lambda_{3}}}\left\langle\Delta \lambda_{1},\left[\bar{\lambda}_{2}, \bar{\lambda}_{3}\right] \lambda_{4}\right\rangle-\left\langle\Delta \lambda_{1},\left[\bar{\lambda}_{2}, \bar{\lambda}_{3}\right] \lambda_{4}\right\rangle=0
$$

Here, non-vanishing coefficients $\langle\Delta \alpha, \beta \gamma\rangle$ and $\langle[\delta, \epsilon], \bar{\beta}\rangle$ applied in the first and third lines of $H$ induce a commutator $\left[p^{\alpha} q^{\beta} q^{\gamma}, p^{\delta} p^{\epsilon} p^{\beta}\right] \hbar^{0}$, whereas applied twice in the second line induce the commutator $(-1)^{n+1}\left[p^{\alpha} p^{\bar{\beta}} q^{\gamma}, p^{\delta} p^{\epsilon} q^{\bar{\beta}}\right] \hbar^{0}$.

The sixth vanishes due to Jacobi:

$$
\left\langle[\cdot, \cdot]^{2}\left(\lambda_{1}, \lambda_{2}, \lambda_{3}\right), \lambda_{4}\right\rangle=0 .
$$

- The coefficients in front of terms of the form $p^{\lambda_{1}} q^{\lambda_{2}} \hbar^{2}$ are given by

$\left\langle\Delta \lambda_{1}, x \lambda_{2}\right\rangle \cdot\langle\Delta x, y \bar{y}\rangle+\left\langle\Delta \lambda_{1}, x y\right\rangle \cdot\left(\sum_{Z_{x, y}}\left\langle\Delta x, \bar{y} \lambda_{2}\right\rangle+\left\langle[x, y], \lambda_{2}\right\rangle\right)$.

The sum of the first two expressions vanishes due to co-Jacobi. The third expression, $[\cdot, \cdot] \circ \Delta\left(\lambda_{1}\right)$, vanishes by the involutive property of the Lie bi-algebra. 
- The coefficients in front of terms of the form $p^{\lambda_{1}} p^{\lambda_{2}} \hbar^{2}$ are given by

$$
\begin{aligned}
& \left(\left\langle\left[\lambda_{1}, \lambda_{2}\right], x\right\rangle+\sum_{Z_{\lambda_{1}, \lambda_{2}}}\left\langle\Delta \lambda_{1}, \bar{\lambda}_{2} x\right\rangle\right) \cdot\langle\Delta x, y \bar{y}\rangle \\
& +\sum_{Z_{\lambda_{1}, \lambda_{2}}}\left\langle\Delta \lambda_{1}, x y\right\rangle \cdot\left(\left\langle[x, y], \bar{\lambda}_{2}\right\rangle+\left\langle\Delta \lambda_{2}, \bar{x} \bar{y}\right\rangle\right. \\
& \left.\quad+\sum_{Z_{x, y}}\left(\left\langle\left[x, \lambda_{2}\right], \bar{y}\right\rangle+\left\langle\Delta x, \bar{y} \bar{\lambda}_{2}\right\rangle\right)\right) \cdot
\end{aligned}
$$

The sum of the first and fifth expressions vanishes by compatibility. The second vanishes by co-Jacobi. The third vanishes by involutivity. The fourth vanishes by symmetry. The sixth vanishes by co-Jacobi.

- The coefficients in front of terms of the form $p^{\lambda_{1}} p^{\lambda_{2}} p^{\lambda_{3}} p^{\lambda_{4}} \hbar^{1}$ are given by

$$
\begin{aligned}
& \sum_{Z_{4 C 2}}\left(\sum_{Z_{\lambda_{1}, \lambda_{2}}}\left\langle\Delta \lambda_{1}, \bar{\lambda}_{2} x\right\rangle+\left\langle\left[\lambda_{1}, \lambda_{2}\right], x\right\rangle\right) \cdot\left(\left\langle\Delta x, \bar{\lambda}_{3} \bar{\lambda}_{4}\right\rangle+\left\langle\left[\lambda_{4}, \lambda_{3}\right], \bar{x}\right\rangle\right. \\
& \left.\quad+\sum_{Z_{\lambda_{3}, \lambda_{4}}}\left(\left\langle\Delta \lambda_{3}, \bar{x} \bar{\lambda}_{4}\right\rangle+\left\langle\left[x, \lambda_{3}\right], \bar{\lambda}_{4}\right\rangle\right)\right) .
\end{aligned}
$$

Here the sum is over the six ways of separating the four $\lambda_{i}$ into two groups of two. The summand as written shows $\lambda_{1}, \lambda_{2}$ separated from $\lambda_{3}, \lambda_{4}$.

We have $4+4=8$ expressions to consider. The 12 terms in the first expression vanish as 4 triples by co-Jacobi. The second and seventh expressions add up to 24 terms which re-arrange to make 12 canceling pairs such as

$$
\left\langle\Delta \lambda_{1}, \bar{\lambda}_{2} \bar{x}\right\rangle\left\langle\Delta \lambda_{3}, \lambda_{4} x\right\rangle-\left\langle\Delta \lambda_{1}, \bar{\lambda}_{2} \bar{x}\right\rangle\left\langle\Delta \lambda_{3}, \lambda_{4} x\right\rangle=0 .
$$

This may again be seen by looking at non-vanishing coefficients $\langle\Delta \alpha, \bar{\beta} \gamma\rangle$ and $\langle\Delta \delta, \bar{\epsilon} \bar{\gamma}\rangle$, which in the second and the third line of $H$ give both $\left[p^{\alpha} p^{\beta} q^{\gamma}, p^{\delta} p^{\epsilon} p^{\gamma}\right] \hbar^{0}$ and $(-1)^{n+1}\left[p^{\alpha} p^{\beta} p^{\bar{\gamma}}, p^{\delta} p^{\epsilon} q^{\bar{\gamma}}\right] \hbar^{0}$.

The third expression vanishes on its own by a similar symmetry. The sum of the fourth and fifth expression vanishes by compatibility. The sixth expressions vanishes on its own by symmetry. The eighth expression vanishes by Jacobi.

We end with two conjectures. 
Conjecture 4.3. The quasi-isomorphism type of the induced quantum $L_{\infty}$ structure from $H^{\prime}$ on $X$ is independent of the choice of representatives used to generate $H_{*}\left(\Omega^{\mathrm{eq}}(X), K\right)$, and hence is a manifold invariant.

Conjecture 4.4. The quasi-isomorphism type of the induced quantum $L_{\infty}$ structure from $H^{\prime}$ is the same as the one defined by counting some moduli space of curves, such as the SFT H.

Proposition 4.1 implies the second conjecture when $X$ is a surface with a hyperbolic metric. Following our earlier discussion, part of the attempt would involve defining string topology operations on the Morse complex generated by the oriented geodesics.

\section{References}

[1] S. Barannikov and M. Kontsevich, Frobenius manifolds and formality of Lie polyvector fields, Internat. Math. Res. Notices. 4 (1998) 201-215,

[2] M. Chas and D. Sullivan, String topology, math.GT/9911159.

[3] K. Cieliebak and J. Latschev, The role of string topology in symplectic field theory, ar $\chi$ iv:0706.3284.

[4] K. Cieliebak, T. Ekholm, J. Latschev and L. Ng, in preparation.

[5] G. Drummond-Cole, J. Terilla and T. Tradler, Algebras over cobar(coFrob), arXiv:0807.1241v1.

[6] Y. Eliashberg, A. Givental and H. Hofer, Introduction to symplectic field theory, in 'GAFA 2000 (Tel Aviv, 1999)', Geom. Funct. Anal., Special Volume, Part II, 560-673, 2000.

[7] M. Gromov, Pseudoholomorphic curves in symplectic manifolds, Invent. Math. 82(2) (1985), 307-347.

[8] J.-S. Park, Flat family of QFTs and quantization of d-algebras, arXiv:hep-th/0308130.

[9] J. Terilla, Smoothness theorem for differential BV algebras, J. Topology 1(3) (2008), 693-702.

[10] J.-S. Park, J. Terilla and T. Tradler, Quantum backgrounds and QFT, arXiv:math/ 0605459 .

[11] D. Sullivan and M. Sullivan, Open string topology and classical knots, in preparation.

UNIVERSITY OF MASSACHUSETTS

AmHerst, MA 01002

E-mail address: sullivan@math.umass.edu

Queens College

City University of New York

FLushing, NY 11367

E-mail address: jterilla@qc.cuny.edu

NYC College of Technology

City University of New York

Brooklyn, NY 11201

E-mail address: ttradler@citytech.cuny.edu 
Received 10/05/2007, accepted 07/01/2008

The first author was supported in part by NSF DMS-0707091.

The second and third authors were supported in part by a CUNY Collaborative Incentive Research Award.

The authors wish to thank the Einstein Chair at the CUNY-Graduate Center for hosting them during this collaboration. They also wish to thank Janko Latschev for explaining his work, and Jae-Suk Park and Mahmoud Zeinalian for many helpful insights and discussions. The third author would like to acknowledge the Max Planck Institute in Bonn for its support. 
